\title{
Experimental measurements of a prototype high-concentration Fresnel lens and sun-tracking method for photovoltaic panel's efficiency enhancement
}

\author{
Meraj Rajaee $^{1} \cdot$ Seyed Mohamad Bagher Ghorashi ${ }^{1}$
}

Received: 27 December 2014/ Accepted: 12 June 2015/Published online: 27 August 2015

(C) The Author(s) 2015. This article is published with open access at Springerlink.com

\begin{abstract}
Concentrator photovoltaic modules are a promising technology for highly efficient solar energy conversion. This system presents several advantages due to additional degrees of freedom that has been provided by the spectral separation such as cost and mass reduction, increase in the incident solar flux on PV cells and performances. This paper has proposed a unique photovoltaic solar cell system that consists of semi-Fresnel lens convergent structure and a novel two axis sun tracking module to enhance the efficiency of solar cell by using less cell area and energy losses. The grooves of this lens are calculated according to the refraction and convergent angles of the light easy for perpendicular incidence angle. The update time interval during tracking causes misalignment of the lens' optical axis versus the sunrays. Then an inventive sun-tracking method is introduced to adjust the module so that the incident rays are always perpendicular to the module's surface. As a result, all rays will be refracted with the predetermined angles. This way the focus area is reduced and smaller cells can be used. We also mentioned different module connections in order to provide compensation method during losses, for networks and power systems. Experimental results show that using semi-Fresnel lens, along with the sun-tracking method increases the efficiency of PV panel.
\end{abstract}

Keywords Concentrator - Semi-Fresnel - Photovoltaic . Efficiency $\cdot$ Tracking method

Seyed Mohamad Bagher Ghorashi

mghorashi@kashanu.ac.ir

1 Department of Laser and Photonics, University of Kashan, Kashan, Iran

\section{Introduction}

PV concentrator systems reduce the area of photovoltaic cells required, increase the solar radiation intensity on the photovoltaic cells and can lead to a reduction in total system cost, if the costs of the concentrator and tracking assembly are less than that of the area of photovoltaic cells displaced.

Although they are mainly composed of silicon, which is a widespread material in nature, the process and thus the final product is expensive. Many investigations have been done to raise the performance of PV cells and reduce the required cell area and its final cost. The proposed methods are mainly based on concentrating the sunrays on a small cell area. There are several concentrator technologies that include parabolic trough solar concentrator, linear refractive Fresnel lens, linear reflective Fresnel lens, dish-shaped reflectors, etc. [1, 2]. Compared to other concentrator technologies, Fresnel lens is characterized by some advantages such as small volume, low weight, short focus, high efficiency, and low cost that motivate researchers to design and commercialize Fresnel-based solar concentrators $[3,4]$. With the improvements of lens material since the official invention of Fresnel lens in 1822, especially polymethylmethacrylate (PMMA), which has a good transmissivity and resistance to sunlight, interest in Fresnel lenses for concentrated solar energy applications rose in the latter half of the twentieth century. The recent developments of concentrated solar energy applications using Fresnel lens systems include imaging Fresnel lens solar concentration systems and non-imaging systems [5]. Researches have revealed that it is the refractive surface of a conventional lens which defines its focusing properties. Based on this fact the Fresnel lens was invented. Later, researchers found out that using Fresnel lens as a 
concentrator in PV systems can effectively increase the system's efficiency. A concentrating photovoltaic (CPV) system with a steady electric output adopting an electrically tunable concentration ratio of the liquid crystals (LC) lens is demonstrated [6]. The module consists of a Fresnel lens array (including 16 Fresnel lenses, each $0.120 \mathrm{~m} \times 0.120 \mathrm{~m}$ ), a solar cell array (including 16 solar cells connected in series, each $0.025 \mathrm{~m} \times 0.025 \mathrm{~m}$ ) which was pasted onto an aluminum plate heat sink and below the Fresnel array. The authors pointed out that using a Fresnel lens array, instead of a large Fresnel lens, can reduce the thermal energy of the solar cells. Zhai et al. [1] experimentally investigated a concentrating solar collector using linear Fresnel lens. Test results revealed that the indication of lost energy was $0.578 \mathrm{~W} / \mathrm{m}^{2} \mathrm{~K}$, which was much smaller than solar collectors without concentrator. In [1], authors used five small Fresnel lenses made of polymethyl, evacuated tube absorber, stepping motor, etc. as the main parts of the module. The module was equipped with a suntracking system that tracked the sunlight and a $20 \mathrm{~mm}$ light strip was produced. The advantages of this solar collector are: (1) low cost due to accessibility of polymethyl and evacuated glass tube, (2) the area of receiver solar cells is smaller than modules without concentrator, (3) the module is easy to deploy in large scale at high temperature [1]. Rai et al. [9] have investigated the Fresnel lens application for space photovoltaic. The authors proposed the optimum linear Fresnel lens concentrator in terms of concentration ratio. Shvartz and Soluyanov [8] presented space Fresnel concentrated modules with GaInp/GaAs/Ge triple-junction (TJ) solar cells. The module included 4 linear Fresnel lenses, 4 linear photo receivers containing $12 \mathrm{TJ}$ solar cells, and a copper heat sink. Optical characteristics of the linear Fresnel lens were estimated accordingly [8]. Wagner et al. [9] have proposed a refractive system, which enhances solar system's efficiency and reduces its cost. The proposed model works based on separating sunlight (white light) into different wavelengths. Then using a Fresnel lens and with the help of progresses in semiconductors, each wavelength can be concentrated on a different material to be absorbed effectively. This will increase the solar system's efficiency. A switchable Fresnel zone plate lens is demonstrated using a polymer-stabilized liquid crystal. The fabrication process is relatively simple and the device can be operated below $10 \mathrm{~V}$ with fast response time [10]. In this paper, a novel refractive structure, named semi-Fresnel lens, is proposed. The proposed model works by calculating the groove angles of Fresnel lens based on the refraction and convergent angles of the orthogonal incident ray. To be clearer, we assume that the incoming ray is directly refracted and converged to a special point. Then using Snell's law and simple trigonometry we find these refraction and convergent angles. Finally based on these two parameters, we calculate the groove angles. This method ensures that every incoming ray will refract directly with refraction angle calculated before. As a result all rays will be converged to a predetermined point with a special convergent angle. Therefore, the needed solar cell area can be significantly reduced. It should not be left unmentioned that, since all calculations are based on the incidence angle orthogonal to the lens surface, the most efficiency will be obtained when the Sun is exactly above the module. To provide this situation in practice, we suggest a sun-tracking method which tracks the sun and adjusts the module so that the incidence angle is always 90 degrees. This way, all incoming rays are concentrated to a line resulting in producing a 2-cm light strip. This will reduce the needed solar cell area resulting in lower cost and lower loss of energy. Based on this assumption a solar panel containing four rows of $2 \mathrm{~cm} \times 90 \mathrm{~cm}$ solar cells is designed and implemented. A Fresnel lens panel including four $90 \mathrm{~cm} \times 20 \mathrm{~cm}$ semi-Fresnel arrays is placed on a the solar cell module at a distance equal to its focal length which is $5 \mathrm{~cm}$. Test results show that the designed model effectively increases the efficiency of the PV system, reducing its cost and loss of energy [7].

\section{Designing the model}

\section{The theory behind the proposed approach}

Centuries ago, it was recognized that the contour of the refracting surface of a conventional lens defines its focusing properties. The bulk of material between the refracting surfaces has no effect (other than increasing absorption losses) on the optical properties of the lens [8].

Much research has been carried out to improve the performance of the Fresnel lens in concentrating the sunlight, which will result in a smaller focus area. As a result, smaller solar cells can be used. These methods also tried to reduce the losses of Fresnel lenses. Here we consider two main losses that result from spherical aberration and total internal reflection (TIR). For lenses made with spherical surfaces, rays which are parallel to the optical axis, but at different distances from the optical axis, fail to converge at the same point. This is called spherical aberration. When moving from a denser medium into a less dense one (i.e., $n 1>n 2$ ), above an incidence angle known as the critical angle, all light is reflected. This phenomenon is known as TIR (later we will discuss how TIR affects our designed model). The critical angle is approximately 41.5 degrees for Plexiglas in air $[9,10]$.

Although some researchers have tried to use TIR to reduce the spherical aberration and reduce the focus area [11], we prefer to eliminate TIR by designing the groove 
angles based on the convergent angle $\alpha$ so that all the incoming rays can be refracted directly to the focus point. This way the loss of energy of the light is considerably reduced. $\alpha$ (for incidence angle orthogonal to the surface of the lens) is derived by using simple trigonometry. Then we can find $\theta 2$ (the refraction angle) and $\theta$ (the groove angle) accordingly. Therefore, we can make sure that for the orthogonal incident rays there will be no spherical aberration. Using this method for calculating the grooves' angles, the only problem is in keeping the incidence angle orthogonal to the lens which will be done by using a suntracking system.

\section{Calculating the groove angles}

The proposed structure for the semi-Fresnel lens is shown in Fig. 1. As it can be seen from the figure, the lens is flat on one side and has fine grooves on the other side. Our purpose is to concentrate all incoming rays to the central line in the focus of the lens. Assuming the lens focal length to be $f$, we will derive the angle of the grooves based on Snell's law. We will follow the derivation for the right groove. We name the angle of this groove with the horizontal direction to be $\theta$. Assume the light beam makes the angle of $\theta_{1}$ with the orthogonal line to the groove (Fig. 1) and it refracts with an angle of $\theta_{2}$ with the same orthogonal line. This exit beam will finally make angle $\alpha$ with the focal line. Therefore, we can write Snell's law for this optical beam:

$n_{1} \sin \theta_{1}=n_{0} \sin \theta_{2}$

Again referring to Fig. 1 , the $\theta$ and $\theta_{1}$ angles have both axes orthogonal to each other and thus based on simple geometrical laws they are equal:

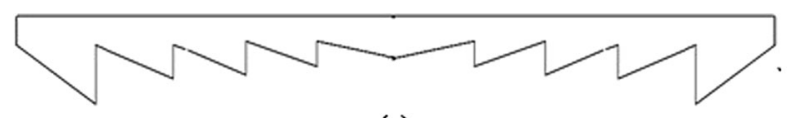

(a)

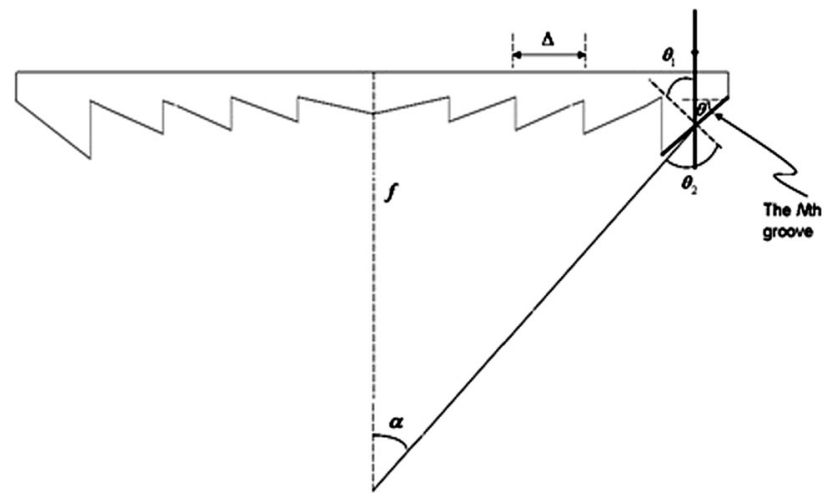

Fig. 1 The proposed semi-Fresnel lens. a The whole structure of the lens. b Extracting the groove's angle $\theta_{1}=\theta$

On the other side, by extending the incident beam we see that the angle $\theta_{2}$ is divided into two angles, one equal to $\theta_{1}$ (and thus $\theta$ ) and the other one equal to $\alpha$ which is again clear from the figure. This yields the following relation:

$\theta_{2}=\theta+\alpha$

Using Eqs. (2) and (3), Eq. (1) can be rewritten in the following way:

$n_{1} \sin \theta=n_{0} \sin (\theta+\alpha)$

Thus $n 0$ in this equation is the refractive index of the air and can be set equal to 1 . Parameter $n 1$ is the refractive index of the medium where for Plexiglas is around 1.49. The only remaining parameter to be determined for the Eq. (4) to yield the value of the groove's angle $\theta$, is $\alpha$. This angle could be simply calculated from simple trigonometric relations. The sine of this angle is the ratio of the groove distance from the center of the lens and its distance from the focal line:

$$
\begin{aligned}
& \sin \alpha=\frac{(N-1+N / 2) \Delta}{\sqrt{((N-1+N / 2) \Delta)^{2}+f^{2}}} \Rightarrow \\
& \alpha=\sin ^{-1}\left(\frac{(N-1+N / 2) \Delta}{\sqrt{((N-1+N / 2) \Delta)^{2}+f^{2}}}\right)
\end{aligned}
$$

where $\Delta$ is the width of each groove and $N$ is the number of grooves counting from the center part. By determining $\alpha$ from Eq. (4), the groove's angle $\theta$ could be extracted using Eq. (4).

Table 1 represents the angles $\alpha, \theta 2$, and $\theta$ for the grooves on the right side of the Fresnel lens. The angles will be the same for the left side grooves.

As it is shown in Table 1, $\theta$ is calculated for each groove based on the convergent angle $(\alpha)$ of that groove for orthogonal incident ray. It means all rays with nonorthogonal angles will be considered as loss. To be more clear, when the incidence angle is non-orthogonal to the lens's surface one of these situations will occur: TIR or spherical aberration. The reason is that every change in incidence angle will make changes to the angles $\theta 1, \theta 2$ and $\alpha$, which in turn enlarge the focus area of the Fresnel lens.

Table 1 Values of the refraction, convergent, and groove angles

\begin{tabular}{llll}
\hline Groove/angles & $\alpha$ & $\theta 2$ & $\theta$ \\
\hline GCR (center-right) & 11.33 & 31.79 & 20.46 \\
G1R & 21.82 & 54.81 & 32.99 \\
G2R & 30.9 & 69.5 & 38.6 \\
G3R & 38.68 & 79.5 & 41 \\
G4R & 44.42 & 86 & 41.5 \\
\hline
\end{tabular}


Since our purpose is to reduce the solar cell area, we should prevent the focus area of the Fresnel lens from being changed. To do this, we used a sun-tracking system that determines the position of the sun periodically and adjusts the module so that the incidence angle is always orthogonal to the lens's surface.

\section{Constructing the model}

In this section the results of experimental implementation is presented. Figure $4 \mathrm{a}$ shows the used configuration to test the performance of the proposed concentrating structure. As it is obvious in the Fig. 4a, a Fresnel lens panel including four $90 \mathrm{~cm} \times 20 \mathrm{~cm}$ semi-Fresnel lens arrays is placed on a PV module in a distance equal to its focal length which is $5 \mathrm{~cm}$. On the solar panel we have placed four narrow cell rows with a width of $2 \mathrm{~cm}$, equal to the width of light strip resulting from the lens. The solar panel is shown in Fig. 2. Figure 3 shows our proposed semiFresnel lens made of Plexiglas.

\section{Experimental results}

In this section the results of experimental implementation are presented. Figure 4 shows the used setup to test the performance of the proposed concentrating structure. As it is obvious in Fig. 4, a Fresnel lens panel including four $90 \mathrm{~cm} \times 20 \mathrm{~cm}$ semi-Fresnel arrays is placed on a solar panel at a distance equal to its focal length which has a value of $5 \mathrm{~cm}$. On the solar panel, we have placed four narrow cell rows with a width of $2 \mathrm{~cm}$, equal to the concentrating area of the lens.

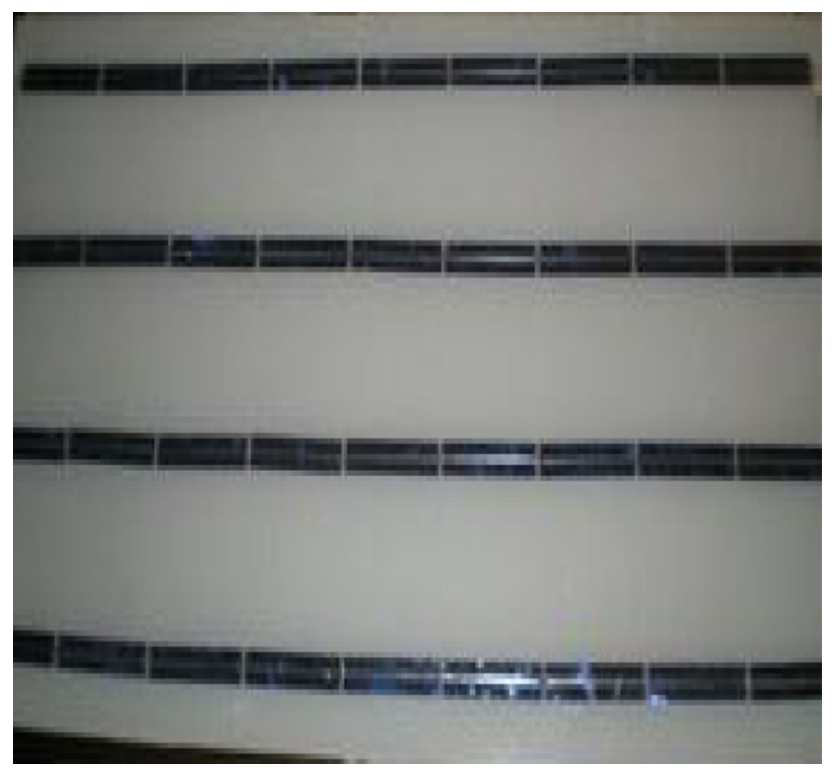

Fig. 2 Solar panel

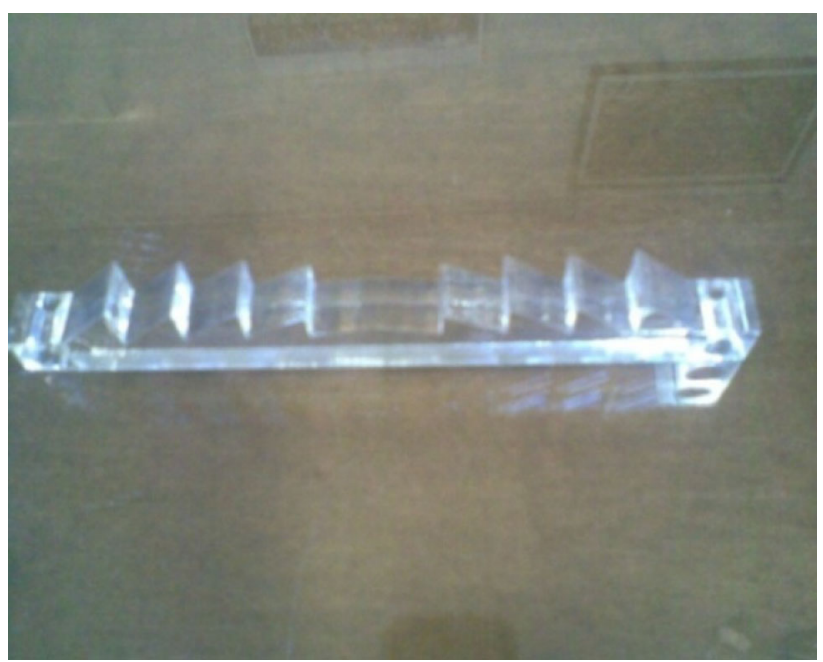

Fig. 3 A single semi-Fresnel lens

To show the superiority of the proposed lens structure in improving the efficiency of PV panel, we have done the same experiment with a conventional Fresnel lens.

The output power versus derived voltage for different amount of incident energy per unit area (in lux) is plotted in Fig. 5. As is clear from the curves, the peak power for different amount of incident energies per unit area is higher for the proposed semi-Fresnel lens compared to the conventional Fresnel lens.

\section{Sun-tracking system method}

As mentioned above, we first calculate the convergent angle of the incoming light and then the groove angles are determined accordingly. The mathematical analyses given above have been obtained for the perpendicular incidence angle. But in practice because of the sun's movement during the day, we cannot have the orthogonal incidence angle during the entire daytime. Based on this fact and in order to observe the impact of the orthogonal incidence angle on the performance of PV panel, we manually set the panel so that the orthogonal incidence angle was obtained for the entire daytime.

What we have used is a two-axis sun-tracking system. Single-axis tracking systems are considerably cheaper and easier to construct, but their efficiency is lower than that of two-axis sun-tracking systems. Two-axis sun-tracking systems can be applied in all types of solar systems to increase their efficiency [12].

The two-axis tracking system could be an azimuth-elevation tracking or polar tracking. In azimuth-elevation tracking, which is the most common method used in large solar dish systems, the dish rotates in a plane parallel to the earth (azimuth) and in another plane perpendicular to it 
Fig. 4 Experimental setup.

a The whole structure for testing the performance of the proposed structure. b Semi-Fresnel lens concentrating the incident radiation on the panel

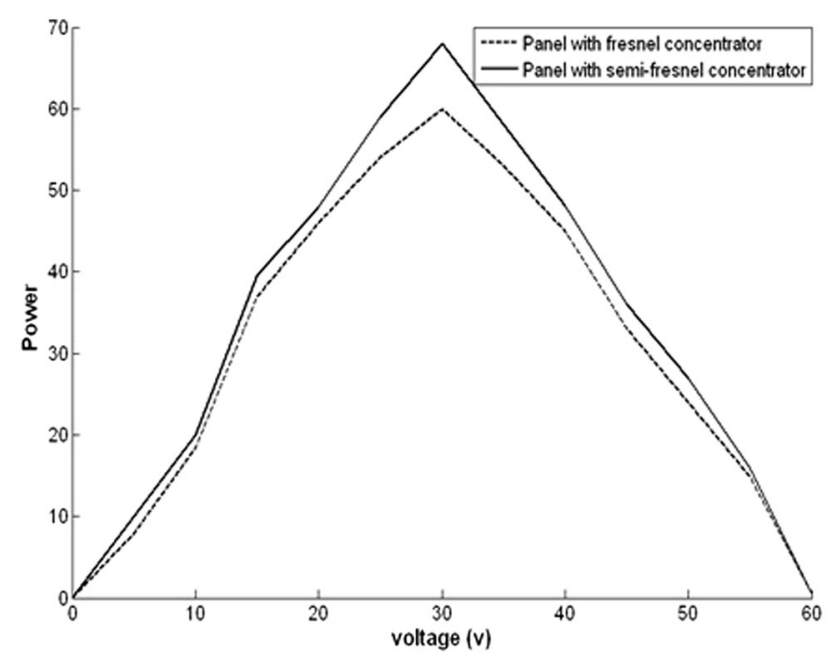

Fig. 5 Power versus voltage for the panel with Fresnel concentrator (dashed line) and semi-Fresnel concentrator (solid line)

(elevation), which gives the collector left-right and updown rotation. Rotational rates can be easily calculated during all day. In polar tracking, which is the most common method in small solar dish systems, the collector
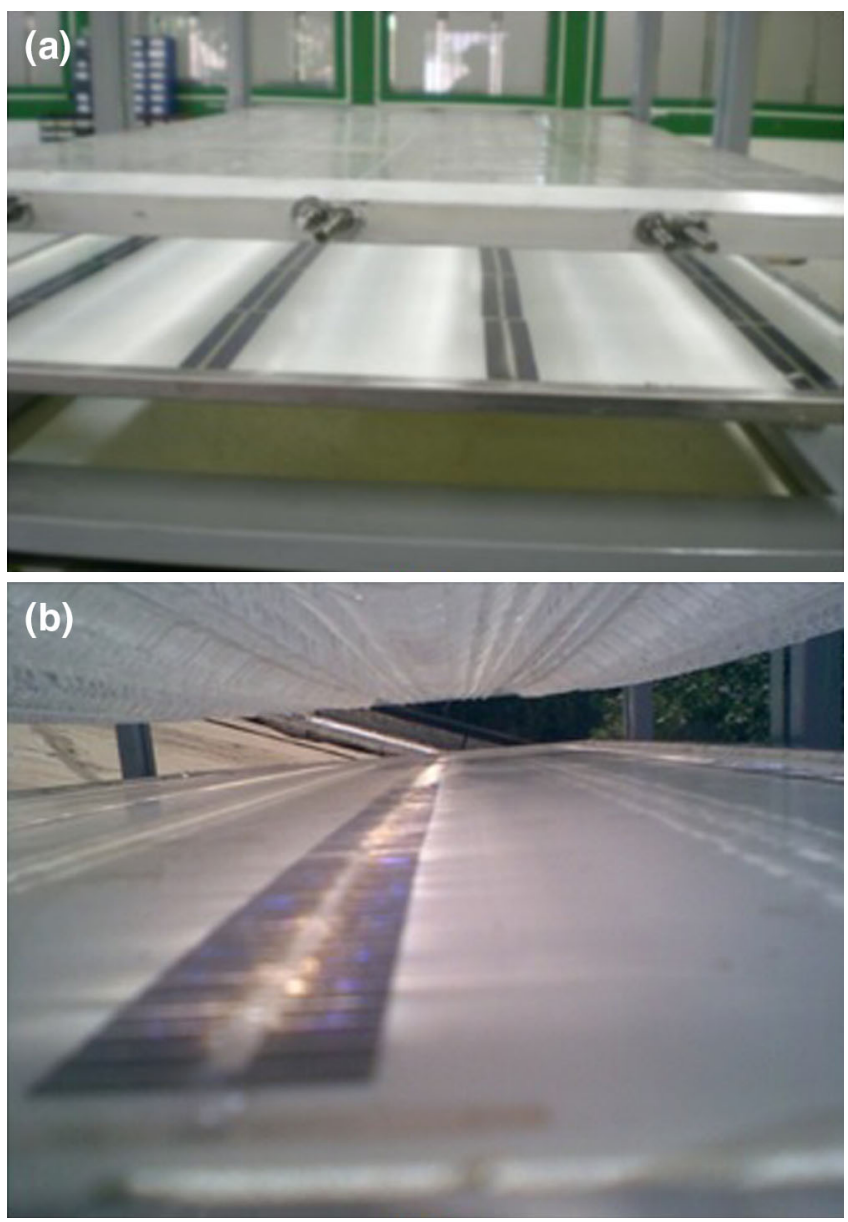

rotates about an axis parallel to the earth's axis of rotation. The collector rotates at a constant rate of $15^{\circ}$ per hour to match the rotational speed of the earth. The other axis of rotation, the declination axis, is perpendicular to the polar axis. Movement about this axis occurs slowly and varies by $\pm 23.5^{\circ}$ over a year [13].

The sun-tracking systems can be realized as closed- or open-loop control systems. The closed-loop systems use photo sensors to position the solar cell modules. Due to permanent position changes, these systems can spend more energy than they gain, especially when the weather is changing. The other possibility is to use open-loop systems where different algorithms provide pre-defined trajectories for the tracking systems. Their trajectories can be accurately determined because the relative position of the sun can be precisely calculated at any time for any location on the earth. The hybrid control systems combine the openloop tracking trajectories based on the solar movement models and the closed-loop control, using dynamic feedback controllers [14].

Many investigations have been carried out to design and employ two-axis sun-tracking systems; however, only a few were cited in the literature that investigated the effect 
of using two-axis sun-tracking systems controlled by modern, computerized control systems, such as the programmable logic controller (PLC) control system. AbuMalouh et al. [12] proposed a spherical solar cooker design and construction using two-axis sun-tracking system to control the spherical solar cooker rotation. This suntracking system depends on PLC and frequency control. It assures that the sunlight beam is normal to the dish at any time of the day.

Al-Mohamad [15] presented a sun-tracking design, whereby the movement of a photovoltaic module was controlled to follow the sun's radiation using a PLC unit. A PLC unit was employed to control and monitor the mechanical movement of the PV module and to collect and store data related to the sun's radiation. It also allows the connection of many PV modules in series or parallel connection. It is found that the daily output power of the PV was increased by more than $20 \%$ in comparison with that of a fixed module and exceeded it by at least $40 \%$ for the period of spanning morning and evening hours. The PV tracking system can be employed as a stand-alone device and it could be connected to a personal computer through the RS232 serial port to monitor the whole process on a computer screen. This technique reduces the costs of tracking systems and makes it a cost-effective technology.

Tejwany and Solanki [16] designed a novel mechanism of sun tracking with automatic cleaning of PV modules and cleaning mechanism of the PV module consists of sliding brushes, which slides over module and cleans it twice a day, wherein PV panel makes a rotation of $360^{\circ}$ in a day. It is observed that the daily energy generation of a flat $\mathrm{PV}$ module (which is kept on the ground) increases the efficiency to $15 \%$ for single axis tracking.

Seme et al. [14] proposed a new method for prediction of the time-dependent solar radiation on a tilted surface for the two-axis sun-tracking system for a PV system. The trajectories of the sun-tracking system are determined in an optimization procedure. It is applied in the new procedure for determining the tilt and azimuth angle trajectories, which assures the maximum energy production in the PV system, considering the energy consumed in the tracking system. Their method is general and gives the optimal results for the applied solar radiation prediction and the tracking system model. Generality of the proposed method remains even in the case when the applied tracking system model and the prediction of the solar radiation are replaces with the more advanced ones.

Hiramatsu et al. [17] developed a two-axis tracking apparatus, which was put into operation with non-imaging Fresnel lens. The excellent tracking ability was verified by yielding results in all three tracking performance evaluations.
Radhi Mahmood and Muhammad [18] designed and implemented a smart relay-based sun-tracking systems to keep the PV array perpendicular to the sun during the day hours. The smart relay-based controller (SR3B261BD) was used to control the motion of the two-axis sun-tracking surface. Preliminary measurements indicated that the use of the two-axis sun tracker would increase daily energy $42 \%$ more than the stationary solar panel and $1 \%$ more than the single axis. The designed system operated smoothly with precise positioning about $7.5^{\circ}$ per half hour.

Chong and Wong [19] derived a novel on-axis general sun-tracking formula and integrated it into an open-loop azimuth-elevation sun-tracking system for improving the tracking accuracy. The installation defect of a $25 \mathrm{~m}^{2}$ solar concentrator prototype had induced significant sun-tracking errors, range from 12.12 to $17.54 \mathrm{mrad}$. With the use of a CCD camera for recording the solar image, three misaligned angles from ideal azimuth-elevation axes have been determined and corrected by changing of the general formula parameters' values to better the tracking accuracy to $2.99 \mathrm{mrad}$. Further fine-tuning has been made to attain the tracking accuracy up to $0.96 \mathrm{mrad}$, by including the motor step count. The general formula has been demonstrated to be capable of achieving high tracking accuracy, cost-effectively without complicated sun-tracking solutions.

Figure 6 shows the output power of panel with and without sun-tracking method. As it is obvious in Fig. 6, tracking the sun to adjust the panel, significantly increased the output power of our PV system. Hence, we propose a two-axis open-loop automatic sun-tracking system, the flowchart of which is given in Fig. 7.

Our proposed two-axis sun-tracking algorithm works based on calculations of the incidence angle according to

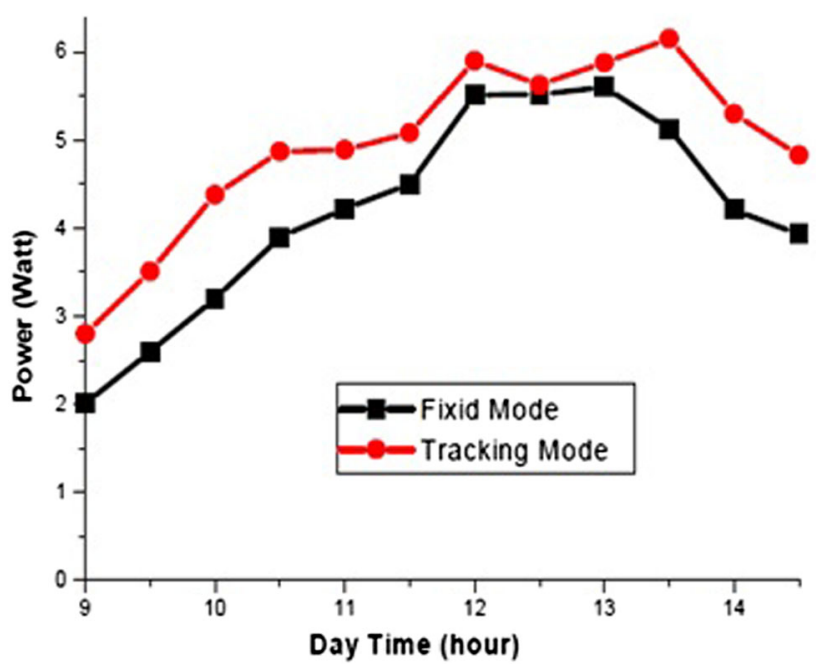

Fig. 6 Output power of PV system with and without sun tracking 
date, time, and geographical position of panel (which means latitude, longitude, and zenith. The sun-tracking system (shown in Fig. 7) is set to start working at sunrise by a 24-hour timer. According to different reasons in defined and predetermined time, the command box starts working and based on the specified time, enables the motor and other components of the system to move. Thus, by rotating the main shaft which is done by an electromotor force, ratchet shaft will also revolve from east to west. In this status, two contrived gears on top of the ratchet shaft are unitized separately that will cause the panel moving from up to down and vice versa. Therefore the panel moves in two axes from east to west and up to down and vice versa. According to the calculated time and order of the command box, the panel moves towards the sun during the day. At the end of the day, using the contrived buttons while finishing the task of the system and pushing keys, the device will automatically turn to its initial dawn position and make it ready to work for the next day. Note that this system is able to rotate the panel $180^{\circ}$ from sunrise to sunset (from east to west) during the day and $90^{\circ}$ from morning to noon (from down to up) and $90^{\circ}$ from noon to evening (from up to down) to move it completely towards the sun so that can make the panel benefit maximum form the incidence time of sunlight.

\section{System components}

This system is composed of four parts:

1. Base

2. Mechanical or rotator part

3. Propellant force or motor

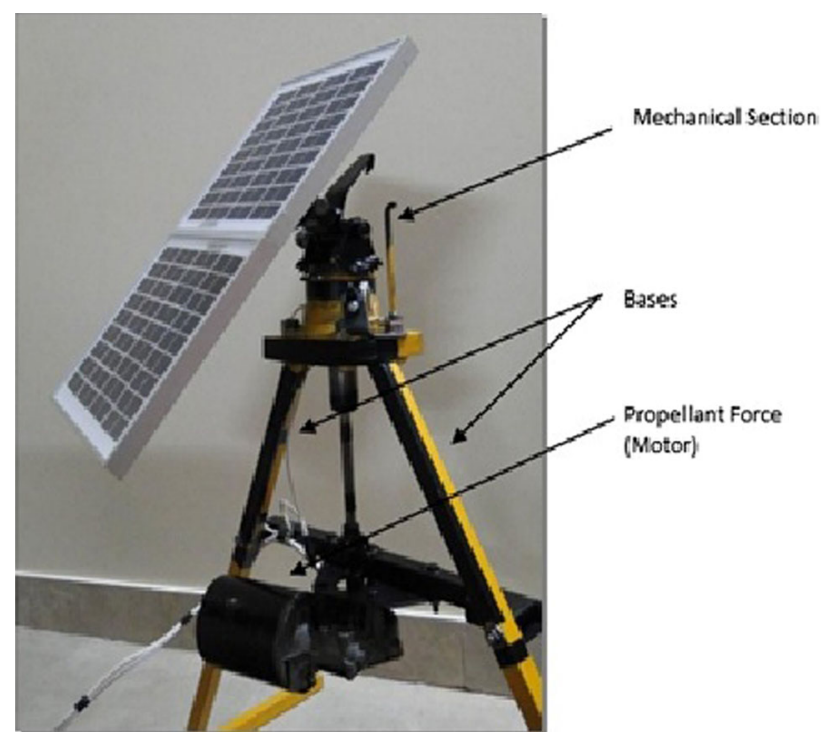

Fig. 7 Schematic of sun-tracking system and solar PV module

\section{Command motor.}

As shown in Fig. 8, the semi-Fresnel lens is placed above the solar cell which the concentrator area is efficiently reduced. The optical segments are implemented on top of the two-axis open-loop sun-tracking system, which automatically controls the rotation of the module in order to have the orthogonal incidence angle.

\section{Connection system}

There are three kinds of connection systems for PV module. These systems are chosen for different applications based on their energy production. Here we will discuss them and for this paper we use off-grid system but PV modules with Fresnel-based refracting structure and suntracking method can be applied with grid-connected systems on order to compensate loses in power lines and networks.

A stand-alone system does not have a connection to the electricity mains (grid). Stand-alone systems vary widely in size and. If the load is to be supplied independently of solar insulation, the generated power is stored and buffered with a battery. In non-portable applications where weight is not an issue, lead acid batteries are most commonly used for their low cost and tolerance for abuse. A charge controller may be incorporated in the system to: (a) avoid battery damage by excessive charging or discharging and, (b) optimizing the production of the cells or modules by maximum power point tracking (MPPT). However, in simple

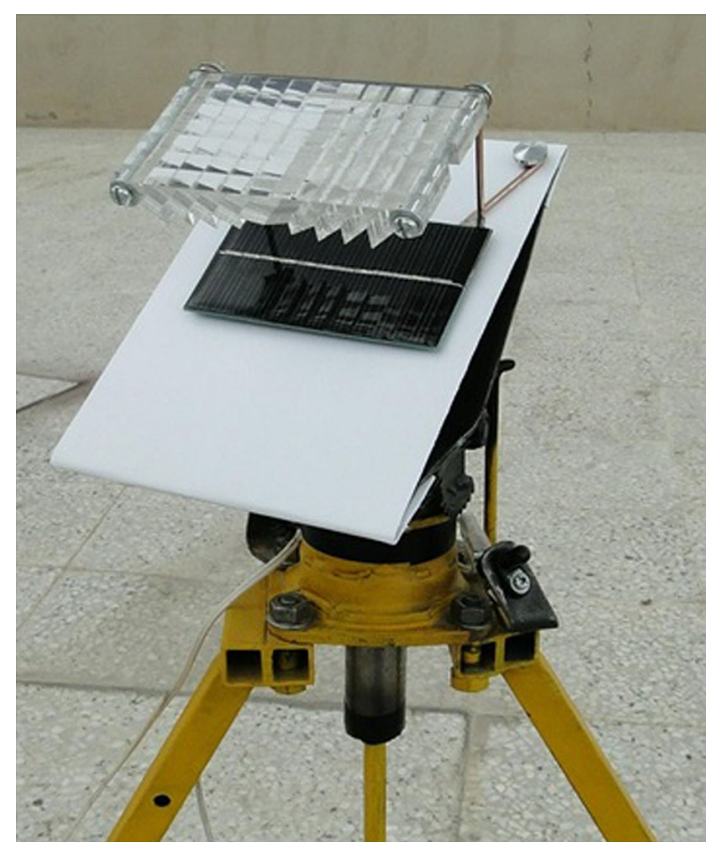

Fig. 8 Implementation of the system 
PV systems where the PV module voltage is matched to the battery voltage, the use of MPPT electronics is generally considered unnecessary, since the battery voltage is stable enough to provide near-maximum power collection from the PV module (Fig. 9).

A grid-connected system is connected to a large independent grid (typically the public electricity grid) and feeds power into the grid. Grid-connected systems vary in size from residential $(2-10 \mathrm{kWp})$ to solar power stations (up to $10 \mathrm{~s}$ of MWp). This is a form of decentralized electricity generation. In the case of residential or building mounted grid-connected PV systems, the electricity demand of the building is met by the PV system. Only the excess is fed into the grid when there is an excess. The feeding of electricity into the grid requires the transformation of $\mathrm{DC}$ into $\mathrm{AC}$ by a special, grid-controlled solar inverter [20]. In $\mathrm{kW}$ sized installations the DC side system voltage is as high as permitted (typically $1000 \mathrm{~V}$ ) to limit ohmic losses. Most modules (72 crystalline silicon cells) generate about $160 \mathrm{~W}$ at $36 \mathrm{~V}$. It is sometimes necessary or desirable to connect the modules partially in parallel rather than all in series [20].

A hybrid system combines PV with other forms of generation, usually a diesel generator. The other form of

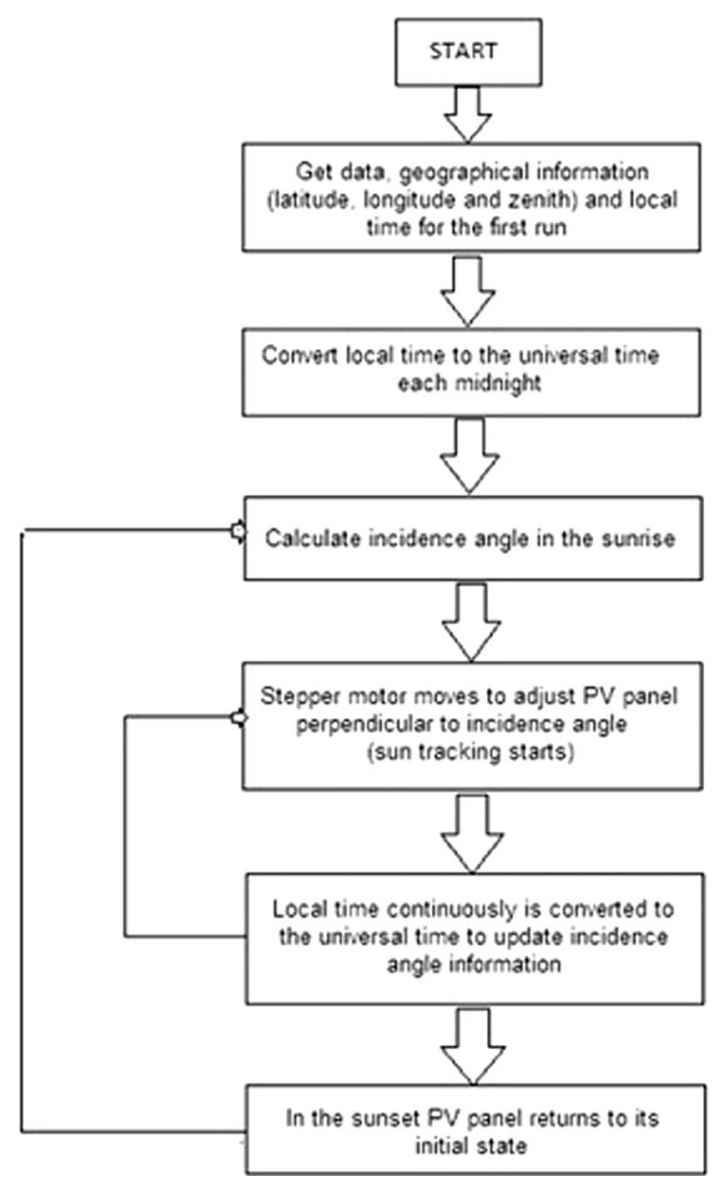

generation may be a type able to modulate power output as a function of demand. However more than one renewable form of energy may be used e.g. wind. The photovoltaic power generation serves to reduce the consumption of non renewable fuel. Hybrid systems are most often found on islands.

\section{Conclusion}

In this paper we proposed a new concentrating structure for improving the performance of photovoltaic cells. To design the proposed semi-Fresnel lens, we used the simple trigonometry to determine the refraction and convergent angles of orthogonal incident ray. Then using Snell's law we determine the groove angle for each groove. As in practice the most efficiency is obtained for the orthogonal incident ray; we use a two-axis open-loop sun-tracking system to keep the module in a position at which the incoming ray is always orthogonal to the lens surface. This way the effects of TIR and spherical aberration in scattering the rays are removed. The outstanding feature of this concentrator is its lower loss and aberration compared to conventional structure with Fresnel lens. It also reduces the amount of solar cell needed to receive the sunlight. The refractive structure was tested experimentally with a narrow PV cell and the output power was calculated for different derived voltages. The resultant values were compared with the similar values for the PV cell with Fresnel concentrator. The results confirmed the superiority of the proposed method.

In future works and studies we can extend the system with proper connection method such as hybrid system. This will demonstrate higher performance for power systems and facing losses in networks while using modules connected together and working with another source of energy with respect to the pre-defined application.

We would also add automatic cleaning device -as there is space between the panel and Fresnel lens, the dust accumulated on the surface of the panel will affect the efficiencyin order to clean the panels regularly in order to enhance the efficiency by $15 \%$ and shows higher performance in longterm usage and the automatic system is needed in cases where there is less physical access to panels.

Open Access This article is distributed under the terms of the Creative Commons Attribution 4.0 International License (http://crea tivecommons.org/licenses/by/4.0/), which permits unrestricted use, distribution, and reproduction in any medium, provided you give appropriate credit to the original author(s) and the source, provide a link to the Creative Commons license, and indicate if changes were made.

Fig. 9 Flowchart of sun-tracking system 


\section{References}

1. Zhai, H., Dai, Y.J., Wu, J.Y., Wang, R.Z., Zhang, L.Y.: "Experimental investigation and analysis on a concentrating solar collector using linear Fresnel lens". Energy Convers. Manag. 51, 48-55 (2010)

2. Mo, S., Chen, Z., Hu, P.: "Performance of a passively cooled fresnel lens concentrating photovoltaic module. 3rd World Conference on Photovoltaic Energv Conversion", Osaka, Japan, pp. 2379-2382, May 2003

3. O'Neill, M.J., Piszczor, M.F., Eskenazi, M.I., McDanal, A.J., George, P.J., Botke, M.M., Brandhorst, H.W., Edwards, D.L., Hoppe, D.T.: Ultra-light stretched fresnel lens solar concentrator for space power applications. International symposium on optical science and technology, SPIE, No. 5179-17 (2003)

4. Piszczor, M.F., O'Neill, M.J.: Development of a dome fresnel fens/GaAs photovoltaic Cconcentrator for space applications. 19th IEEE-PVSC (1987)

5. Leutz, R., Suzuki, A., Akisawa, A., Kashiwagi, T.: Flux densities in optimum non imaging Fresnel lens solar concentrator for space. 0-7803-5772-8/00/\$10.00 IEEE (2000)

6. Shvartz, M.Z., Chosta, O.I., Grilikhes, V.A., Rumyantsev, V.D., Andreev, V.M.: Space Fresnel lens concentrator modules with triple junction solar cells. 0-7803-8707-4/05/\$20.00, IEEE (2005)

7. Wagner, D., He, L.: An innovative solar system with high efficiency and low cost. 978-1-4244-5892-9/10/\$26.00, IEEE (2010)

8. Shvarts, M.Z., Soluyanov, A.A.: High quality fresnel lenses in a variety of sizes and focal lengths. In: Fresnel Technologies Advances in Science and Technology, Vol. 74, pp 188-195 (2010)

9. Rai, A.K., Kaushika, N.D., Singh, B., Agarwal, N.: Simulation model of ANN based maximum power point tracking controller for solar PV system. Sol. Energy Mater. Sol. Cells 95, 773-778 (2011). doi:10.1016/j.solmat.2010.10.022

10. The Fresnel equations and Brewster law. www.Google.com

11. Omar, A.M., Shaari, S., Omar, A.R., Yahir, M.R.: An automated solar photovoltaic biaxial tracking system. In: First international power and energy conference PECon 2006, Malaysia, pp. 44-47 (2006)

12. Abu-Malouh, R., Abdallah, S., Muslih, I.M.: Design, construction and operation of spherical solar cooker with automatic sun tracking system. Energy Convers. Manag. 52, 615-620 (2011)

13. Poullikkas, A., Kourtis, G., Hadjipaschalis, I.: Parametric comparative study for the installation of solar dish technologies in Cyprus. 7th Mediterranean Conference and Exhibition on Power Generation, Transmission, Distribution and Energy Conversion, paper No. MED10/110, Agia Napa, Cyprus, November 2010

14. Seme, S., Stumberger, G., Vorsic, J.: Maximum efficiency trajectories of a two axes sun tracking systems determined considering tracking system consumption. IEEE Transactions on power electronics, vol. 26, no. 4, April 2011, 0885-8993/\$26.00 IEEE

15. Al-Mohamad, Ali: Efficiency improvements of photovoltaic panels using a sun tracking system. Appl. Energy 79(0306-2619), 345-354 (2004)

16. Tejwany, R., Solanki, C.S.: $360^{\circ}$ sun tracking with automated cleaning system for solar PV modules. In: Photovoltaic specialists conference (PVSC), 2010 35th IEEE. IEEE (2010). doi:10. 1109/PVSC.2010.5614475

17. Hiramatsu, M., Miyazaki, Y., Egarni, T., Akisawa, A., Mizuta, Y.: Development of non-imaging Fresnel lens and sun tracking device. 3rd World Conference on Photovoltaic Energy Conversion, Osaka, Japan, pp. 2383-2385, May 2003

18. Sungur, C.: Multi-axes sun-tracking system with PLC control for photovoltaic panels in Turkey. Renew Energy 34, 1119-1125 (2009)

19. Chong, K.-K., Wong, C.-W.: Open-loop azimuth-elevation sun tracking system using on-axis general sun tracking formula for achieving tracking accuracy of below 1 mrad. 978-1-4244-58929/10/\$26.00 @2010 IEEE

20. How Solar Power Works. http://www.solarcity.com/residential/ how-solar-works.aspx 